\title{
Physical activity, a new norm for mythical virus
}

Haemi Jee (iD https://orcid.org/0000-0002-9066-1472

An era of new norm has suddenly faced the world due to the smallest organism on the planet. Since the first reported outbreak in Wuhan, China in December 2019, the number of exposures has exponentially increased worldwide. The problem is that the number exposed is every rising. This virus has broken many of the known expert knowledge on virus.

Among many of the side effects of coronavirus outbreak, inability to participate in social gatherings and activities have brought another serious problem. Many of the media are reporting on the mental health crisis that includes anxiety and depression with uprising number of suicide world-wide. A period of uncertainty and repeated rise in exposure to virus have exhausted mental as well as physical health. Physical inactivity lead to decline in metabolic and immunological functions among others. Such decline in physiological functions rapidly lead to vicious cycle of physical inactivity and mental health issues.

Extended uncertainty period of coronavirus pandemic has led many people into the state of panic. There should be a contingency plan to relieve build up physical and mental stress instead of suppressing all activities. Physical activity has been known to relieve mental and physical complications that may arise from discontinued daily life activities, sedentary behavior, and inflict negative health consequences. Recent studies report of positive effects and need of exercise intervention for depression during the coro- navirus epidemic.

Many of the studies were conducted to remotely providing exercise training such as home-based or telerehabilitation programs. Although numerous home-based and telerehabilitation programs have been reported to be effective even before the outbreak, the efficacy has been incomparable to actual training programs. More studies should be conducted on such remotely conducted exercise training since physically remoted or isolated exercising practice may become a new norm even long after the corona pandemic. It should be the role and responsibility of the scholars to provide more effect and safe ways to maintain health even during the period of crisis. The Journal of Exercise Rehabilitation is an ideal platform to share and provide vital information to the public during the unprecedent era.

\section{CONFLICT OF INTEREST}

No potential conflict of interest relevant to this article was reported.

Department of Physical Therapy, Namseoul University 91 Daehak-ro, Seonghwan-eup, Sebuk-gu, Cheonan 31020, Korea 